\title{
TIPOS DE MARKETING EN COMERCIOS TEXTILES DE LA GUAJIRA COLOMBIANA
}

\author{
Meudis Quintero Ramos ${ }^{1 *}$ y Norelys Cárdenas Fragozo ${ }^{2}$ \\ ${ }^{1,2}$ Universidad de La Guajira. Maicao, Colombia \\ *Autor de correspondencia: meudys1012@hotmail.com
}

Recibido Mayo 2020; Aceptado Julio 2020

\begin{abstract}
Resumen - Se buscó establecer los tipos de marketing, en los comerciantes del sector de ropa en el municipio de Maicao, norte de Colombia. La presente investigación se enmarcó dentro de un enfoque positivista, diseño de campo, no experimental, transeccional, descriptivo. Así mismo, se realizó un censo poblacional con sesenta y tres (63) sujetos, sector Cacaíto y sector el Triángulo del municipio de Maicao. Se realizó una encuesta, con alternativas de respuesta tipo escala Likert las cuales se le asignó una valoración numérica de uno (1) a cinco (5) según el escalamiento representado de la siguiente manera: Siempre (5); Casi Siempre (4); Algunas Veces (3); Casi Nunca (2) y Nunca (1). Se concluye que los tipos de marketing comercial, social y de causa generan rentabilidades en las ventas de prendas de vestir, satisfaciendo a clientes y sus diferentes entornos.
\end{abstract}

Palabras clave: Mercadeo; frontera, textiles, comercio; La Guajira.

\section{TYPES OF MARKETING IN TEXTILE STORES IN THE GUAJIRA REGION OF COLOMBIA}

\begin{abstract}
It was sought to establish the types of marketing, in the merchants of the clothing sector in the municipality of Maicao, northern Colombia. This research was framed within a positivist approach, field design, non-experimental, transectional and descriptive. Likewise, a population census was carried out with sixty-three (63) subjects, in the Cacaíto sector and the Triangle sector of the municipality of Maicao. A survey was carried out, with Likert scale response alternatives, which were assigned a numerical rating of one (1) to five (5) according to the scale represented as follows: Always (5); Almost Always (4); Sometimes (3); Almost Never (2) and Never (1). It is concluded that the types of commercial, social and cause marketing generate profits in garment sales, satisfying customers and their different environments.
\end{abstract}

Keywords: Marketing, border, textiles, trade, La Guajira.

\section{Introducción}

En la actualidad, las actividades comerciales han venido posicionándose lentamente y cada vez con mayor fuerza ya que este sector genera un dominio muy importante en la economía de los países. Por estas y más razones es arduo el trabajo del marketing, ya que forja a que las organizaciones se vuelvan más competitivas, además la globalización interviene en ellas, para que estas se adapten al cambio no solo en su imagen ni en sus estrategias, si no también que se adapten a un mercado que cada vez va en crecimiento (Briceño et al., 2019). 
Es por ello, que las últimas disciplinas de la administración de las organizaciones es el Marketing lo cual es utilizada en los procesos de comercialización determinadas en una serie de actividades destinadas a satisfacer las necesidades y deseos de los proveedores, comerciantes y vendedores lo cual traerá beneficios para las organizaciones que ponen en prácticas estas actividades; por estas razone se puede decir que el marketing es indispensable para el logro de las metas propuesta de mercadeo en el mundo globalizado (Gómez \& Pérez, 2018; González \& Plaza, 2017). La mercadotecnia a nivel globalizado, es de suma importancia para estas empresas multinacionales ya sean granes o pequeñas debido que están enmarcadas al reconocimiento comercial en aras de satisfacer a los clientes y proveedores en cuanto a los productos y/o servicios en el mercado global, acarreando mayor rentabilidad en los costes y mejorar en eficiencia, eficacia y productividad, para las organizaciones que usen las estrategias de marketing como elemento fundamental para sobrevivir en el mundo global (Alvarado et al., 2016).

En Colombia, el comportamiento de la economía desde el decenio de los 90 puede caracterizarse por un crecimiento moderado, aceptable y constante, una reducción gradual de la inflación, un sector externo dinámico, un nivel relativamente alto de reservas internacionales, un sistema financiero definitivamente más sólido que el promedio latinoamericano, avances en la infraestructura física, particularmente en telecomunicaciones, algunos avances en educación, en cobertura de salud y seguridad, y una balanza corriente deficitaria pero financiada con capitales de largo plazo (Romero et al., 2016; Alarcón-Chávez \& Granda-García, 2018). Ahora bien, en la zona fronteriza de Colombia, específicamente en el municipio de Maicao, se puede decir que el mismo es un puerto terrestre libre de Régimen Aduanero Especial, creado por el decreto 1706 de 1992, ratificado en el Estatuto Aduanero (Decreto 2685 de 2000), modificado por el Decreto 1187 de 2000 y elevado de jerarquía mediante la Ley 677 del 03 de agosto de 2001 (Por la cual se expiden normas sobre tratamientos excepcionales para regímenes territoriales) (Quintero et al., 2018; Muñoz et al., 2016).

Existen diversos tipos de marketing para que las empresas puedan señalar la existencia de la organización para realizar acciones pertinentes dentro de ellas. De esta manera, para la presente investigación se tendrán en cuenta: el marketing comercial, social y de causa bajo el constructo de Saldarriaga et al. (2016), con la intencionalidad de reconocer el contexto de mercado. Según Ruiz et al. (2020), indica que los tipos de marketing son "agrupados de maneras diferentes y estos pueden variar aunque se refieran a lo mismo. Por lo general, consiste en un conjunto de estrategias y posibilidades de diferentes tipos". Son los distintos tipos de marketing que se desarrollan para cada segmento de mercado, producto y servicio. Para los autores Quintero \& Morillo (2017), este se refiere a todo aquello que es susceptible por lo que esto incluye bienes, servicios e ideas. De ahí que los posibles tipos de aplicación del marketing sean muy variados y este pueda emplearse no solo en empresas con actividad comercial, sino en cualquier organización en la que se dé un proceso de intercambio. Según lo postulados por los autores antes mencionados en los párrafos anteriores se diferencian distintos tipos de marketing como se verán a continuación: Marketing Comercial, Marketing Social y Marketing De Causa [9]. Teniendo como referencia todo lo hasta aquí expuesto, en este trabajo se busco establecer los tipos de marketing, en los comerciantes del sector de ropa en el municipio de Maicao, Norte de Colombia.

\section{Materiales y Métodos}

El enfoque de la investigación es positivista debido a que se orienta a la realidad desde el punto de vista objetivista, el tipo de investigación es descriptivo, diseño de campo, no experimental, Transaccional. La población objeto de estudio está conformada por los comerciantes de prendas en el municipio de Maicao,

IPSA SCIENTIA - Vol. 5 No $1-2020$ 
Departamento de la Guajira, se realizó un censo poblacional con sesenta y tres sujetos (63), sector Cacaíto y sector el Triángulo del municipio de Maicao, como se muestra en la tabla 1.

Tabla 1: Muestra poblacional

\begin{tabular}{|c|c|c|c|}
\hline Sector & Descripción & Ubicación & Total \\
\hline Cacaíto & Locales de ropas entre almacenes y colmenas & Calle 11 & 36 \\
\hline Triangulo & Locales de ropas entre almacenes y colmenas & Calle 14 & 27 \\
\hline \multicolumn{2}{|r|}{} \\
\hline
\end{tabular}

Fuente: Elaboración propia

La recolección de la información se realizó con base en la técnica observación directa mediante encuesta y se seleccionó como instrumentos un cuestionario. Se midió con un instrumento escala tipo Likert. La escala de opciones vino dada por las siguientes alternativas de respuestas las cuales se le asignó una valoración numérica de uno (1) a cinco (5) según el escalamiento de Likert representado de la siguiente manera: Siempre (5); Casi Siempre (4); Algunas Veces (3); Casi Nunca (2) y Nunca (1).

Los datos tabulados se organizan en tablas en las cuales se recogen los resultados estadísticos del procesamiento frecuencial y porcentual de las cifras aportadas por los participantes del estudio con el objeto de tener una visión sintética y precisa de aquellos. Adicionalmente, los resultados estadísticos se ilustran a través de gráficos de barra, realizados en un sistema de estadísticas llamado IBM® ${ }^{\circledR}$ SPSS Statistics ${ }^{\circledR} 2015$ como principal software estadístico que ofrece técnicas de recolección de datos y analítica para solucionar el problema planteado de investigación objeto de estudio.

\section{Resultados y Discusión}

Los resultados revelados en el tabla 2, muestran que el mayor porcentaje se encuentra en la alternativa de siempre con un 28,5\% lo que indica según la ponderación del baremo que está en una categoría alta, mostrando que el desarrollo de actividades se encuentran concerniente a la variable de estudios.

Tabla 2: Resultados de la dimensión Tipos de Marketing

\begin{tabular}{|c|c|c|c|c|c|c|c|c|c|c|c|c|c|}
\hline \multirow{2}{*}{ Dimensión } & \multirow{2}{*}{ Indicador } & \multicolumn{2}{|c|}{ Siempre } & \multicolumn{2}{|c|}{ Casi siempre } & \multicolumn{2}{|c|}{ Algunas veces } & \multicolumn{2}{|c|}{ Casi nunca } & \multicolumn{2}{|c|}{ Nunca } & \multirow[b]{2}{*}{ Media } & \multirow[b]{2}{*}{ Desviación } \\
\hline & & $\mathrm{Fa}$ & $\%$ & $\mathrm{Fa}$ & $\%$ & $\mathrm{Fa}$ & $\%$ & $\mathrm{Fa}$ & $\%$ & $\mathrm{Fa}$ & $\%$ & & \\
\hline \multirow{4}{*}{ Tipos } & $\begin{array}{l}\text { Marketing } \\
\text { comercial }\end{array}$ & 18 & 28,5 & 14 & 22,2 & 13 & 20,6 & 12 & 19,0 & 6 & 9,5 & 3,4 & 1,2 \\
\hline & $\begin{array}{c}\text { Marketing } \\
\text { social }\end{array}$ & 20 & 31,7 & 14 & 22,2 & 18 & 28,5 & 6 & 9,5 & 4 & 6,3 & 3,6 & 1,1 \\
\hline & $\begin{array}{l}\text { Marketing } \\
\text { de causa }\end{array}$ & 8 & 12,6 & 11 & 17,4 & 26 & 41,2 & 10 & 15,8 & 7 & 11,1 & 3,0 & 1,1 \\
\hline & Promedio & 15 & 24,3 & 13 & 20,6 & 19 & 30,1 & 9 & 14,8 & 6 & 9,0 & 3,4 & 1,1 \\
\hline
\end{tabular}

Fuente: Elaboración propia

En este mismo sentido, se palpa que los comerciantes en las ventas de prendas de vestir crean identidad de marca con la cual el cliente se puede relacionar, informan a los individuos sobre los beneficios que puede recibir personalmente de consumir un producto y por tanto educan a los consumidores sobre asuntos sociales colectivos. 
Seguidamente, se puede observar que el 22,2 \% contesto casi siempre, 20,6\% contesto algunas veces, el 19,0\% casi nunca y el 9,5 \% nunca la media de este indicador 3,4 aquí se evidencia una tendencia moderada por parte del personal entrevistado y la desviación fue de 1,2 indicando que hay dispersión muy alta y por ende, no hay homogeneidad en las alternativas de respuestas suministrada por los informantes claves.

De acuerdo a Flores (2013), el marketing comercial, cuya finalidad es la detección de necesidades mal atendidas o insatisfechas, las cuales al ser cubiertas van a generar ingresos destinados a cubrir las utilidades y los costos de la empresa para que ésta se desarrolle en un entorno competitivo y de libre mercado. El objetivo del marketing comercial es lograr los márgenes de utilidades para la empresa mediante la satisfacción de las necesidades de los clientes. Su finalidad es la generación de utilidades mediante la satisfacción de las necesidades de los clientes.

En el indicador marketing social los expuestos en el tabla 2 revelan que 31,7\% de los entrevistados respondieron siempre, mientras que un $22,2 \%$ casi siempre, un $28,5 \%$ en algunas veces, $9,5 \%$ en casi nunca y un 6,3\% en siempre. Indicando este que el de mayor incidencia fue siempre queriendo manifestar según la ponderación del baremo que se encuentra en una categoría alta dejando ver que llevan a cabo el desarrollo de actividades concerniente a la variable de estudio.

Dentro de esta perspectiva se desarrolla siempre en este indicador se asocia una marca a una buena causa mediante la dedicación de recursos económicos, suelen hacer las compañías que necesitan mejorar su imagen afectada y consiguen lavar su imagen interna consiguiendo a veces mayor motivación en sus empleados. También hay que indicar que existe una tendencia alta en el indicador marketing social con una media de 3,6 y la desviación de 1,1 indicando que existe dispersión muy alta en los datos dados por los informantes claves.

Desde la postura de la investigadora, se considera que hay de inseguridad en las respuestas presentadas por los encuestados porque a pesar que los comerciantes están de acuerdo con la teoría planteada por Mata (2010), el marketing social se orienta a la necesidad social. Las necesidades en los individuos se modifican de manera constante, por tal motivo el bienestar de la sociedad se debe buscar de igual manera. El marketing social se enfoca en buscar un cambio en la sociedad que proporcione mejores condiciones de vida a la comunidad. La finalidad de este marketing es lograr el beneficio de la población.

Por último, se obtienen los datos estadísticos del indicador Marketing de causa, donde se evidencia que el $12,6 \%$ contestaron que siempre, $17,4 \%$ casi siempre, $41,2 \%$ algunas veces, $15,8 \%$ casi nunca y $11,1 \%$ nunca, indicando que en este indicador el de mayor incidencia fue algunas veces, con el $41,2 \%$ de las respuestas dadas por los encuestados, dando entender que según la ponderación del baremo se encuentra en la categoría medio queriendo significar con esto que desarrollan las actividades con debilidades con relación a la variable de estudio.

En todo caso, también hay que mencionar que la media fue de 3,0 indicando que esta se encuentra de manera moderada según las respuestas de los informantes y la desviación es de 1,1, indicando que esta se encuentra alta por tal motivo no se evidencia que no hay respuestas uniforme conforme a lo que respondieron los informantes. En otras palabras la dispersión se aleja del centro los valores de la distribución de las consultas realizadas a los sujetos encuestados. Así mismo, la media del promedio 
general 3,4 y la desviación de 1,1 y de dispersión muy alta donde se indica cómo se alejan los datos respecto de la media aritmética.

Existe incertidumbre en las respuestas desplegadas por los encuestados muestra de ello se está de acuerdo con la teoría planteada por Torres (2013), donde manifiesta que el marketing de causas, las empresas del sector privado contribuyen con causas sociales, siempre y cuando estas generen beneficios para las mismas. El objetivo del marketing de causas es el de ayudar y contribuir con la sociedad pero sin descuidar la ganancia de utilidades de las empresas, su finalidad es generar beneficios para la empresa y la sociedad (Cepeda-Palacio, Velásquez-Estrada \& Marín-Gómez, 2017).

\section{Conclusiones}

Se puede concluir que los comerciantes entre los tipos de Marketing comercial, social y de causa para generar rentabilidades en las ventas de prendas de vestir y de esta manera se han logrado satisfacer a los clientes y sus diferentes entornos. Estos tipos de estrategias son usados para identificar las necesidades de vender en el comercio, y sobre todo lo que el público desea. De esta manera se es más efectivo para alcanzar los objetivos de ventas a la hora de ofrecer valor al objetivo de venta en el mercado. Por tanto, en esta dimensión tipos de marketing se definen estrategias que le permiten establecerse en el mercado laboral. En este caso es necesario recomendar, que conozcan las necesidades, deseos y preferencias de los consumidores en las últimas tendencias del mercado para entrar en competencia con el mercado global.

Se realicen planes comerciales donde se determinen las políticas comerciales para alcanzar los objetivos propuestos y las líneas de acciones, las reglas y directrices comerciales, utilizar campañas publicitarias para prendas de vestir para damas y caballeros que permitan producir utilidades para el negocio. Una imagen corporativa aliada con los comerciantes. Generar ventas al por mayor y el reconocimiento de marca. Facilitar la penetración de nuevos mercados.

\section{Referencias}

Alarcón-Chávez, C. R., \& Granda-García, M. I. (2018). El marketing y la fidelización empresarial como apuesta estratégica para pymes en Ecuador. Dominio de las Ciencias, 4(1), 131-140.

Alvarado, Y. N. R., Cuenta, M. M. C., \& Castellar, L. S. A. (2016). Marketing mix y el enfoque de valor como estrategia competitiva en el sector turístico del departamento de La Guajira-Colombia. Red Internacional de Investigadores en Competitividad, 9(1), 964-979.

Briceño, L., Ruiz, M., Villalba, V., Castro, A., Vidal, C. \& Severiche, C. (2019). Elementos de la gerencia del siglo XXI: Una fundamentación desde la bioética y el liderazgo. En V. Meriño (Ed.), Gestión del Conocimiento Perspectiva Multidisciplinaria (Vol. 12, pp. 277-295). Fondo Editorial Universitario de la Universidad Nacional Experimental Sur del Lago Jesús María Semprúm.

Cepeda-Palacio, S. D., Velásquez-Estrada, L. J. \& Marín-Gómez, B. E. (2017). Análisis evaluativo a los procesos de marketing en la internacionalización de las pequeñas y medianas empresas de alimentos de Medellín. Estudios Gerenciales, 33(144), 271-280.

Flores, R.D. (2013). Incorporación del marketing dentro de un sistema de gestión de calidad. Su evolución histórica dentro de las normas de calidad. Visión Gerencial, (1), 23-44.

IPSA SCIENTIA - Vol. $5 \mathrm{~N}^{\circ} 1-2020$ 


\section{Tipos de marketing en comercios textiles de la Guajira Colombiana}

Gómez, M. C. O. \& Pérez, W.G. (2018). Communication as a Marketing Strategy: Cross-cultural Analysis of Young People from Mexico and Colombia Based on Demographic Segmentation. Mediterranean Journal of Social Sciences, 9(3), 173.

González Romo, Z. \& Plaza Romero, N. (2017). Estrategias de marketing digital en el sector de la moda de lujo. Interacción y redes sociales como herramienta necesaria Revista Académica sobre Documentación Digital y Comunicación Interactiva, $15,17-27$.

Mata, M. R. (2010). Mercadeo social, responsabilidad social y balance social: conceptos a desarrollar por instituciones universitarias. Telos, 12(1), 29-42.

Muñoz, D., Martelo, R. \& Ruiz, M. (2016). Políticas económicas e interculturalidad en La Guajira colombo-venezolana. IPSA SCIENTIA: Revista Científica Multidisciplinaria, 1(1), 18-30.

Quintero, M, \& Morillo, E. (2017) Estrategias de marketing empleadas por los comerciantes de prendas de vestir en la "Vitrina Comercial” de Colombia. Neumann Business Review, 3(2), 127-151.

Quintero, M., Morillo, E. \& González, J. (2018). Elementos de la cultura innovativa en el comercio de prendas de vestir del municipio Maicao-Colombia. Neumann Business Review, 4(1), 4-27.

Romero, C., Zúñiga-Toro, G. \& Suárez-Gámez, A (2016). Técnicas de marketing en las empresas de artesanías wayuu en el distrito turístico y cultural de Riohacha, Guajira, Colombia. Omnia, 22(3), 87-99

Ruiz-Cabezas, M.R., Severiche-Sierra, C.A., Briceño-Ariza, L.J. \& Duran-Charris, L.J. (2020). Barreras de competitividad de las PyMEs agrícolas del distrito de Santa Marta (Caribe Colombiano). Revista Espacios, 41(17).

Saldarriaga-Díaz, J.M., Vélez-Zapata, C. \& Betancur-Ramírez, G. (2016). Estrategias de mercadeo de los vendedores ambulantes. Semestre Económico, 19(39), 155-171.

Torres, C. E. T. (2013). El marketing al servicio de las organizaciones del tercer sector. Estudios Gerenciales, 29(129), 386-395. 